\title{
SLIDING MODE CONTROL OF NONLINEAR SYSTEMS WITH TIME-VARYING DELAY
}

\author{
Toshiki Oguchi* Kai Tanaka** \\ * Department of Mechanical Engineering, \\ Graduate School of Engineering, Tokyo Metropolitan \\ University \\ 1-1, Minami-Osawa, Hachioji-shi, Tokyo 192-0397 JAPAN \\ oguchi@control.prec.metro-u.ac.jp \\ ** Ishikawajima-Harima Heavy Industries Co., Ltd. \\ 1, Shin-Nakahara, Isogo-ku, Yokohama JAPAN
}

\begin{abstract}
This paper considers a sliding mode control of multivariable nonlinear systems with time-varying delay. In our previous work, a sliding mode control design method combining with the finite spectrum assignment has been developed. In the design method, the sliding surface is constructed by using a coordinates transformation containing past values of the state variables. In most practical systems, however, it is difficult to identify exactly the length of time-delay. In this paper, we discuss the effect of uncertainties in time-delay and parameters on system's behaviour in the sliding mode and derive a sufficient condition to guarantee asymptotic stability for the systems by using Lyapunov-Razumikhin function approach. Effectiveness of the proposed method is tested by a numerical simulation of continuous stirred-tank reactor system. Copyright (C)2005 IFAC
\end{abstract}

Keywords:

Sliding Mode Control, Nonlinear systems, Time-delay,

\section{INTRODUCTION}

Recently, time-delay systems have attracted a lot of attention from theoretical and practical viewpoints. Time-delay exists in not only the engineering but also biological systems and economical systems. The existence of time-delay usually degrades the control performance and sometimes makes the stabilization of the closed loop system difficult. On the other hand, sliding Mode Control (SMC) (Utkin, 1992; Slotine and Li, 1996) is one of the well-known robust control design methods. The most advantage of the sliding mode control is its inherent insensitivity to uncertainties and disturbances which satisfy a certain structural condition, so called a matching condition. The fundamental idea of sliding mode control is to constrain the system trajectory on a predesigned hyperplane by a switching input. A great deal of SMC controller design methods (Young and Özgüner, 1999; Sira-Ramirez, 1988; Sira-Ramirez, 1989; Perruquetti and Barbot, 2002) for finite dimensional systems have been proposed, and several approach have been developed for time-delay linear or nonlinear systems (Cheres et al., 1989; Choi, 1999; ElKhazaly, 1998; Gouaisbaut et al., 2002; Luo and la Sen, 1993; Shyu and Yan, 1993; Bonnet et al., 1999; Li and Yurkovich, 1999). As a SMC design method for retarded nonlinear systems, Oguchi et al. (Oguchi et al., 2002) have proposed a controller design method based upon the finite spectrum assignment(FSA). In the proposed SMC design procedure, the sliding surface is constructed by us- 
ing a variable transformation introduced into the FSA. Therefore the sliding surface which contains the past values of the state variables and has nonlinearity can be designed as a linear hyperplane with a finite number of dimension, and the system governed on the sliding hyperplane is reduced to a lower dimensional linear system. Most practical systems, however, contain uncertainties in a timedelay and the model parameters.

In this paper, we consider a sliding mode control of uncertain retarded nonlinear systems and discuss the effect of uncertainty in the time-delay. In addition, we derive a sufficient condition to guarantee asymptotic stability for the systems in the sliding mode by using Lyapunov-Razumikhin function approach.

This paper is organized as follows: Section 2 is devoted to give a brief review of the sliding mode controller design method based on the finite spectrum assignment. In Section 3, we discuss the effect of time-delay uncertainty. An illustrative example to verify our result is given in $\S 4$. Finally, concluding remarks follow in $\S 5$.

\section{PRELIMINARY}

\subsection{Sliding Mode Control via FSA Approach}

At the beginning, we briefly introduce a sliding mode control by using the finite spectrum assignment approach. We consider the following multivariable nonlinear systems with constant delays in the state.

$$
\begin{aligned}
\dot{x}(t)= & f\left(x(t), x\left(t-\ell_{f_{1}}\right), \cdots, x\left(t-\ell_{f_{p}}\right)\right) \\
& +\sum_{i=1}^{m} g_{i}\left(x(t), x\left(t-\ell_{g_{1}}\right), \ldots, x\left(t-\ell_{g_{q}}\right)\right) u_{i} \\
x(t)= & \psi(t),-\ell_{\max } \leq t \leq 0
\end{aligned}
$$

where $x \in \mathbb{R}^{n}, u_{i} \in \mathbb{R}, f$ and $g_{i}$ are smooth vector fields with $f(0)=0$ and $g_{i}(0) \neq 0$, respectively, and $\psi(t):\left[-\ell_{\max }, 0\right] \rightarrow \mathbb{R}^{n}$ is a given continuous function. Moreover $\ell_{f_{j}}(t)$ for $j=1, \ldots, p$ and $\ell_{g_{j}}$ $j=1, \ldots, q$ are constant delays.

By applying the pure delay operator $\sigma_{*}: \lambda(t) \rightarrow$ $\lambda\left(t-\ell_{*}\right)$, system (1) can be rewritten by the following equation.

$$
\begin{aligned}
\dot{x}(t) & =\bar{f}(x, \boldsymbol{\sigma})+\sum_{i=1}^{m} \bar{g}_{i}(x, \boldsymbol{\sigma}) u_{i}(t) \\
& \triangleq \bar{f}(x, \boldsymbol{\sigma})+\bar{G}(x, \boldsymbol{\sigma}) \boldsymbol{u}(t)
\end{aligned}
$$

where $\bar{G}: \mathcal{C}\left(\left[-\ell_{\max }, 0\right], \mathbb{R}^{n}\right) \rightarrow \mathbb{R}^{n}$ is defined by $\bar{G}(x, \boldsymbol{\sigma})=\left(\bar{g}_{1}(x, \boldsymbol{\sigma}), \cdots, \bar{g}_{m}(x, \boldsymbol{\sigma})\right), \bar{g}_{i}(x, \boldsymbol{\sigma})=$ $g_{i}\left(x(t), \ldots, x\left(t-\ell_{g_{q}}\right) \cdot 1(t)\right.$ and $\boldsymbol{u}=\left(u_{1}, \cdots, u_{m}\right)^{T}$.

For such a system, we use the following derivative instead of the Lie derivative.
Definition 1. delayed state derivative (Oguchi et al., 1999) Let $\bar{f}(x, \boldsymbol{\sigma}) \equiv f\left(x(t), \ldots, x\left(t-\ell_{f_{k}}\right)\right)$ be an $n$-vector whose components are functions of $x(t)$ and $x\left(t-\ell_{f_{i}}\right), 1 \leq i \leq k, \bar{\phi}(x, \boldsymbol{\sigma}) \equiv$ $\phi\left(x(t), \ldots, x\left(t-\ell_{\phi_{m}}\right)\right), 1 \leq i \leq m$, a real-valued function of $x(t)$ and $x\left(t-\ell_{\phi_{i}}\right), 1 \leq i \leq m$, and $\ell_{f_{i}}$ and $\ell_{\phi_{i}}$ positive time delays. Then the derivative of $\bar{\phi}(x, \boldsymbol{\sigma})$ along $\bar{f}(x, \boldsymbol{\sigma})$ is defined by

$$
L_{\bar{f}} \bar{\phi}(x, \boldsymbol{\sigma}) \stackrel{\text { def }}{=} \frac{\partial \bar{\phi}}{\partial x(t)} \bar{f}+\sum_{i=1}^{m} \frac{\partial \bar{\phi}}{\partial \sigma_{\phi_{i}} x} \sigma_{\phi_{i}} \bar{f}(x, \boldsymbol{\sigma}),
$$

where $\sigma_{f_{i}}: x(t) \mapsto x\left(t-\ell_{f_{i}}\right)$ and $\sigma_{\phi_{i}}: x(t) \mapsto$ $x\left(t-\ell_{\phi_{i}}\right)$ be the pure delay operators.

Note that the argument $(x, \boldsymbol{\sigma})$ of $L_{\bar{f}} \bar{\phi}(x, \boldsymbol{\sigma})$ denotes that $L_{\bar{f}} \bar{\phi}(x, \boldsymbol{\sigma})$ is a function of $x(t)$ and $\boldsymbol{\sigma} x$. For $\gamma \geq 2$, the general form of higher order derivatives can be denoted by $L_{\bar{f}}^{\gamma+1} \bar{\phi}(x, \boldsymbol{\sigma})=$ $L_{\bar{f}} L_{\bar{f}}^{\gamma} \bar{\phi}(x, \boldsymbol{\sigma})$.

If $\ell=0$, the delayed state derivative coincides with the conventional Lie derivative. Throughout this paper, we use notation $L_{\bar{f}} \bar{\phi}$ as the delayed state derivative.

Now, we assume that there exist $m$ functions $\bar{\phi}_{i}(x, \boldsymbol{\sigma})$ and $m$ positive integers $k_{i} \in \mathbb{N}$ for $i=$ $1, \ldots, m$ satisfying the following conditions.

(i) $k_{1}+\cdots+k_{m}=n$

(ii) For any $x, \boldsymbol{\sigma} x \in \mathbb{R}^{n}, L_{\bar{g}_{s}} L_{\bar{f}}^{j} \bar{\phi}_{i}(x, \boldsymbol{\sigma})=0$ where $0 \leq j \leq k_{i}-2$ and $1 \leq i, s \leq m$.

(iii) For $x, \boldsymbol{\sigma} x \in \mathbb{R}^{n}$,

$$
\frac{\partial L_{\bar{f}}^{k_{i}-1} \bar{\phi}_{i}(x, \boldsymbol{\sigma})}{\partial \sigma_{f_{j_{1}}} \cdots \sigma_{f_{j_{k_{i}-2}}} x} \bar{g}_{s}\left(\sigma_{f_{j_{1}}} \cdots \sigma_{f_{j_{k_{i}-2}}} x\right)=0
$$

where $1 \leq j_{1} \leq p, 0 \leq j_{2}, \ldots,{ }^{\forall} j_{k_{i}-2} \leq p$, $i, s=1, \ldots, m$ and $\sigma_{f_{0}}: x(t) \mapsto x(t)$.

(iv) The following matrix $P$ is nonsingular for any $x$ and $\boldsymbol{\sigma} x \in \mathbb{R}^{n}$.

$$
P=\left(\begin{array}{ccc}
\frac{\partial L_{\bar{f}}^{k_{1}-1} \bar{\phi}_{1}}{\partial x(t)} \bar{g}_{1} & \cdots & \frac{\partial L_{\bar{f}}^{k_{1}-1} \bar{\phi}_{1}}{\partial x(t)} \bar{g}_{m} \\
\vdots & \cdots & \vdots \\
\frac{\partial L_{\bar{f}}^{k_{m}-1} \bar{\phi}_{m}}{\partial x(t)} \bar{g}_{1} & \cdots & \frac{\partial L_{\bar{f}}^{k_{m}-1} \bar{\phi}_{m}}{\partial x(t)} \bar{g}_{m}
\end{array}\right)
$$

Then, by applying a variable transformation $z(t)=\Phi(x, \boldsymbol{\sigma})$ :

$$
z=\Phi(x, \boldsymbol{\sigma}) \triangleq\left(\begin{array}{c}
\Phi_{1}(x, \boldsymbol{\sigma}) \\
\vdots \\
\Phi_{m}(x, \boldsymbol{\sigma})
\end{array}\right)
$$

where

$\Phi_{i}(x, \boldsymbol{\sigma})=\left(\begin{array}{c}\bar{\phi}_{i}(x, \boldsymbol{\sigma}) \\ \vdots \\ L_{\bar{f}}^{k_{i}-1} \overline{\bar{\phi}}_{i}(x, \boldsymbol{\sigma})\end{array}\right) \in \mathbb{R}^{k_{i}}$ for $i=1, \ldots, m$

and a feedback: 


$$
\left(\begin{array}{c}
u_{1} \\
\vdots \\
u_{m}
\end{array}\right)=-P^{-1}\left\{\left(\begin{array}{c}
L_{\bar{f}}^{k_{1}} \bar{\phi}_{1}(x, \boldsymbol{\sigma}) \\
\vdots \\
L_{\bar{f}}^{k_{m}} \bar{\phi}_{m}(x, \boldsymbol{\sigma})
\end{array}\right)-\left(\begin{array}{c}
v_{1} \\
\vdots \\
v_{m}
\end{array}\right)\right\},
$$

the system (2) is transformed into the following Burnovskii canonical form.

$$
\dot{z}=\operatorname{diag}\left(A_{1}, \cdots, A_{m}\right) z+\operatorname{diag}\left(b_{1}, \cdots, b_{m}\right) \boldsymbol{v}
$$

where

$$
A_{i}=\left(\begin{array}{cccc}
0 & 1 & \cdots & 0 \\
\vdots & \vdots & \ddots & \vdots \\
0 & 0 & \cdots & 1 \\
0 & 0 & \cdots & 0
\end{array}\right) \in \mathbb{R}^{k_{i} \times k_{i}}, \quad b_{i}=\left(\begin{array}{c}
0 \\
\vdots \\
0 \\
1
\end{array}\right) \in \mathbb{R}^{k_{i}}
$$

As a result, system (2) can be decoupled into $m$ linear subsystems with infinite number of dimension. This control method is called a "finite spectrum assignment for retarded nonlinear systems" (Oguchi and Watanabe, 2000), and it is regarded as an extension of the exact linearization of finite dimensional nonlinear systems (Isidori, 1995; Nijmeijer and van der Schaft, 1990) and the finite spectrum assignment for retarded linear systems which are controllable over the polynomial ring (Morse, 1976; Sontag, 1976).

Now, we show a sliding mode controller design method by using the above-mentioned finite spectrum assignment procedure. Assume that the system (1) can be accomplished a finite spectrum assignment by a static feedback. Then by applying the finite spectrum assignment procedure, system (1) can be decoupled into $m$ subsystems as follows.

$$
\dot{z}^{i}=A_{i} z^{i}(t)+b_{i} v_{i}(t), i=1, \ldots, m
$$

Now we design the following switching surface for each subsystem.

$$
S_{i}(z) \equiv\left(s_{i 1}, \cdots, s_{i k_{i}-1}, 1\right) z^{i}=0
$$

where $z^{i}=\left(z_{1}^{i}, \ldots, z_{k_{i}}^{i}\right)^{T} \triangleq \Phi_{i}(x, \boldsymbol{\sigma})$ and $s_{i j}$ are coefficients of Hurwitz polynomial

$$
\lambda^{k_{i}-1}+s_{i k_{i}-1} \lambda^{k_{i}-2}+\cdots+s_{i 2} \lambda+s_{i 1} .
$$

Note that this switching surface consists of not only the current value of $x$ but also the past values of $x$ in $x$-coordinates. This means that the design problem of infinite dimensional nonlinear manifold can be reduced to a design problem of a linear hyperplane in a finite dimensional space by using this design method.

According to a well known switching condition: $S_{i} \frac{d S_{i}}{d t}<0$, a switching control law is derived as follows. For some $M_{i} \geq 0$,

$$
\left\{\begin{array}{l}
v_{i}(t) \leq-\sum_{j=1}^{k_{i}-1} s_{i j} z_{j+1}^{i}-M_{i}, \text { if } S_{i}>0 \\
v_{i}(t) \geq-\sum_{j=1}^{k_{i}-1} s_{i j} z_{j+1}^{i}+M_{i}, \text { if } S_{i}<0
\end{array}\right.
$$

By applying the above switching law, $z$ reaches the sliding surface $(7)$ and $S_{i}(z(t))=\dot{S}_{i}(z(t))=0$ holds in the sliding mode. The behaviour of the system on the sliding surface is governed by the following $n-m$-th order linear system.

$$
\dot{\tilde{z}}(t)=\operatorname{diag}\left(\tilde{A}_{1}, \cdots, \tilde{A}_{m}\right) \tilde{z}(t)
$$

where $\tilde{z} \triangleq \tilde{\Phi}(x, \boldsymbol{\sigma})=\left(\tilde{\Phi}_{1}^{T}, \cdots, \tilde{\Phi}_{m}^{T}\right)^{T} \in \mathbb{R}^{n-m}$,

$$
\tilde{\Phi}_{i}(x, \boldsymbol{\sigma}) \triangleq\left(\bar{\phi}_{i}, L_{\bar{f}} \bar{\phi}_{i}, \cdots, L_{\bar{f}}^{k_{i}-2} \bar{\phi}_{i}\right)^{T} \in \mathbb{R}^{k_{i}-1}
$$

and $\tilde{A}_{i} \in \mathbb{R}^{\left(k_{i}-1\right) \times\left(k_{i}-1\right)}$ is defined by

$$
\tilde{A}_{i} \triangleq\left(\begin{array}{cccc}
0 & 1 & \cdots & 0 \\
\vdots & \vdots & \ddots & \vdots \\
0 & 0 & \cdots & 1 \\
-s_{i 1} & -s_{i 2} & \cdots & -s_{i k_{i}-1}
\end{array}\right) .
$$

Since $s_{i j}$ are coefficients of Hurwitz polynomial $\lambda^{k_{i}-1}+s_{i k_{i}-1} \lambda^{k_{i}-2}+\cdots+s_{i 2} \lambda+s_{i 1}$, we can show that $\lim _{t \rightarrow \infty} \tilde{z}_{i}(t)=0$ holds. As a result, $z(t)$ converges to zero in $z$-coordinates. Moreover it is proved that there exists unique inverse mapping $x(t)=\Psi(z, \boldsymbol{\sigma})$ of the coordinate transformation $z(t)=\Phi(x, \boldsymbol{\sigma})$ in the similar way as the case of single input systems (Oguchi et al., 2002). Therefore it is guaranteed that $x(t)$ also converges to zero in $x$-coordinates.

\section{TIME-VARYING DELAY SYSTEMS}

Since it is difficult to identify exactly the length of a time-delay, we have to consider the effect of the mismatch of time-delay between real plant and the nominal model. In this section, we consider a sliding mode control of nonlinear systems with time-varying delay in the state. The following retarded nonlinear system with time-varying delay in the state is considered.

$$
\left\{\begin{aligned}
\dot{x}(t)= & f\left(x(t), \cdots, x\left(t-\tau_{f_{p}}(t)\right)\right) \\
& +\sum_{i=1}^{m} g_{i}\left(x(t), \ldots, x\left(t-\ell_{g_{q}}\right)\right) u_{i} \\
x(t)= & \psi(t),-\ell_{\max } \leq t \leq 0
\end{aligned}\right.
$$

where $\tau_{f_{j}}(t)$ for $j=1, \ldots, p$ are bounded timevarying delays, and $\tau_{f_{j}}(t) \in\left[\ell_{j, \min }, \ell_{j, \max }\right]$ but $\tau_{f_{j}}(t)$ themselves are unknown.

Let $\tilde{\sigma}_{f_{j}}$ denote a formal time-varying delay operator such that $\tilde{\sigma}_{f_{j}} \lambda(t)=\lambda\left(t-\tau_{f_{j}}(t)\right)$. Then system (10) can be rewritten as

$$
\dot{x}(t)=\bar{f}(x, \tilde{\boldsymbol{\sigma}})+\bar{G}(x, \boldsymbol{\sigma}) \boldsymbol{u} .
$$

By using system (1) as the nominal model of (10), system (11) can be described by

$$
\begin{aligned}
\dot{x}(t) & =\bar{f}(x, \boldsymbol{\sigma})+\bar{G}(x, \boldsymbol{\sigma}) \boldsymbol{u}+\bar{f}(x, \tilde{\boldsymbol{\sigma}})-\bar{f}(x, \boldsymbol{\sigma}) \\
& \triangleq \bar{f}(x, \boldsymbol{\sigma})+\bar{G}(x, \boldsymbol{\sigma}) \boldsymbol{u}+p(t)
\end{aligned}
$$

where $p(t)=\bar{f}(x, \tilde{\boldsymbol{\sigma}})-\bar{f}(x, \boldsymbol{\sigma})$. Then $p(t)$ can be regarded as a perturbation which is added to 
the nominal system (1). Now we assume that the given nominal model satisfies the condition (i)(iv). Then by applying variable transformation (3) and feedback (4) into system (12), system (11) is transformed into

$$
\begin{aligned}
\dot{z}= & \operatorname{diag}\left(A_{1}, \cdots, A_{m}\right) z+\operatorname{diag}\left(b_{1}, \cdots, b_{m}\right) \boldsymbol{v} \\
& +\left.\left(\frac{\partial \Phi}{\partial x}+\frac{\partial \Phi}{\partial \boldsymbol{\sigma} x} \boldsymbol{\sigma}\right) p\right|_{x=\Psi(z, \boldsymbol{\sigma})} .
\end{aligned}
$$

In addition, by applying a switching control law with $M_{i}$ satisfying

$$
M_{i} \geq \sup _{p}\left|\sum_{j=1}^{k_{i}-1} s_{i j} L_{p} L_{\bar{f}}^{j-1} \bar{\phi}_{i}+L_{p} L_{\bar{f}}^{k_{i}-1} \bar{\phi}_{i}\right| .
$$

system (12) can be governed on the sliding surface.

The behaviour of system (12) in the sliding mode is described by the following reduced system.

$$
\dot{\tilde{z}}=\operatorname{diag}\left(\tilde{A}_{1}, \cdots, \tilde{A}_{m}\right) \tilde{z}+\Delta_{z}(\tilde{z}, \boldsymbol{\sigma})
$$

where $\left.\Delta_{z}(\tilde{z}, \boldsymbol{\sigma}) \triangleq \Delta(x, \boldsymbol{\sigma})\right|_{x=\Psi(z, \boldsymbol{\sigma}), S=0}$ and

$$
\begin{aligned}
\Delta(x, \boldsymbol{\sigma}) \triangleq\left(\frac{\partial \tilde{\Phi}}{\partial x}+\frac{\partial \tilde{\Phi}}{\partial \boldsymbol{\sigma} x} \boldsymbol{\sigma}\right) p & \\
= & \operatorname{col}\left(L_{p} \bar{\phi}_{1}, \cdots, L_{p} L_{\bar{f}_{f}}^{k_{1}-2} \phi_{1}, \cdots,\right. \\
& \left.L_{p} \bar{\phi}_{m}, \cdots, L_{p} L_{\bar{f}}^{k_{m}-2} \bar{\phi}_{m}\right) .
\end{aligned}
$$

This equation means that if all $L_{p} L_{\bar{f}}^{j} \phi_{i}(0 \leq j \leq$ $\left.k_{i}-2,1 \leq i \leq m\right)$ vanish, the behaviour of the system on the sliding surface is invariant with respect to $p(t)$. While, if there exists some integer $j\left(j=0, \ldots, k_{i}-2\right)$ and/or $i(i=1, \ldots, m)$ such that $L_{p} L_{\bar{f}}^{j} \phi_{i} \neq 0$, the reduced system on sliding surface is affected by the term $p(t)$. Concerning the stability of the system affected by the model error $p(t)$, we obtain the following theorem.

Theorem 2. If the following conditions hold, the zero solution of system (12) is asymptotically stable.

a) There exists a positive constant $k$ satisfying

$$
\|\Delta(x, \boldsymbol{\sigma})\| \leq k \sup _{\theta \in[-\bar{\ell}, 0]}\|\tilde{\Phi}(x(t+\theta), \boldsymbol{\sigma})\|
$$

where $\|\cdot\|$ denotes the Euclidean norm.

b) There exist a symmetric positive definitematrix $P$ and a positive-definite matrix $Q$ such that

$$
\begin{aligned}
& 0<k<\frac{\lambda_{m}(Q)}{2 \lambda_{M}(P)} \sqrt{\frac{\lambda_{m}(P)}{\lambda_{M}(P)}} \\
& P A+A^{T} P=-Q
\end{aligned}
$$

where $A \triangleq \operatorname{diag}\left(\tilde{A}_{1}, \ldots, \tilde{A}_{m}\right)$, and $\lambda_{M}(P)$ and $\lambda_{m}(P)$ denote the maximum eigenvalue and the minimum eigenvalue of matrix $P$, respectively.
PROOF. Since $A$ is Hurwitz matrix, equation (16) has a unique solution for $P$ corresponding to each positive-definite matrix $Q$. Let choose a Razumikhin function as

$$
V(\tilde{z}(t))=\tilde{z}^{T}(t) P \tilde{z}(t) .
$$

From the positiveness of matrix $P$, the following inequality holds.

$$
\lambda_{m}(P)\|\tilde{z}(t)\|^{2} \leq V(\tilde{z}(t)) \leq \lambda_{M}(P)\|\tilde{z}(t)\|^{2}
$$

This inequality can be rewritten by

$$
\begin{aligned}
\lambda_{m}(P)\|\tilde{z}(t+\theta)\|^{2} & \leq \tilde{z}^{T}(t+\theta) P \tilde{z}(t+\theta) \\
& \leq \lambda_{M}(P)\|\tilde{z}(t+\theta)\|^{2} .
\end{aligned}
$$

Now, we introduce a continuous and non-decreasing function $\eta(s)$ as $\eta(s) \triangleq \eta s,(\eta>1)$ and consider $\tilde{z}_{t} \triangleq \tilde{z}(t+\theta)$ satisfying

$$
\tilde{z}^{T}(t+\theta) P \tilde{z}(t+\theta)<\eta \tilde{z}^{T}(t) P \tilde{z}(t), \quad \forall \theta \in\left[-\bar{\ell}_{z}, 0\right]
$$

Since $\eta>1$ and (18), it follows that

$$
\eta \tilde{z}^{T}(t) P \tilde{z}(t) \leq \eta \lambda_{M}(P)\|\tilde{z}(t)\|^{2} .
$$

From (19),(20) and (21), we obtain

$$
\sup _{\theta \in\left[-\bar{\ell}_{z}, 0\right]}\|\tilde{z}(t+\theta)\|<\sqrt{\eta \frac{\lambda_{M}(P)}{\lambda_{m}(P)}}\|\tilde{z}(t)\| .
$$

While, condition a) can be rewritten in $z$-coordinates as follows.

$$
\left\|\Delta_{z}(\tilde{z}, \boldsymbol{\sigma})\right\| \leq k \sup _{\theta \in\left[-\bar{\ell}_{z}, 0\right]}\|\tilde{z}(t+\theta)\|
$$

Simple computations lead to the following:

$$
\begin{aligned}
\dot{V}(\tilde{z}(t))= & \tilde{z}^{T}(t)\left(P A+A^{T} P\right) \tilde{z}(t)+2 \tilde{z}^{T}(t) P \Delta_{z}(\tilde{z}, \boldsymbol{\sigma}) \\
\leq & -\lambda_{m}(Q)\|\tilde{z}(t)\|^{2} \\
& +2 \lambda_{M}(P)\|\tilde{z}(t)\| \cdot\left\|\Delta_{z}(\tilde{z}, \boldsymbol{\sigma})\right\| \\
\leq & -\lambda_{m}(Q)\|\tilde{z}(t)\|^{2} \\
& \quad+2 \lambda_{M}(P)\|\tilde{z}(t)\| \cdot k \sup _{\theta \in\left[-\bar{\ell}_{z}, 0\right]}\|\tilde{z}(t+\theta)\| \\
< & \left\{2 \lambda_{M}(P) \cdot k \sqrt{\eta \frac{\lambda_{M}(P)}{\lambda_{m}(P)}}-\lambda_{m}(Q)\right\}\|\tilde{z}(t)\|^{2}
\end{aligned}
$$

Here, if $0<a<b$ and $q$ satisfies $1<q<\left(\frac{b}{a}\right)^{2}$, $0<a<\frac{1}{\sqrt{q}} b$ also holds. Therefore if (15) holds, there exists $\eta>1$ satisfying

$$
0<k<\frac{\lambda_{m}(Q)}{2 \lambda_{M}(P)} \sqrt{\frac{\lambda_{m}(P)}{\eta \lambda_{M}(P)}}
$$

and then $\dot{V}(t)<0$ holds. By applying the Lyapunov-Razumikhin theorem (Niculescu, 2001), we can conclude that the zero solution of system (12) is uniformly asymptotically stable. 


\section{AN EXAMPLE}

We consider a control of a two-stages continuous stirred-tank reactor (CSTR) system (Figure 1). The mathematical model of the system is de-

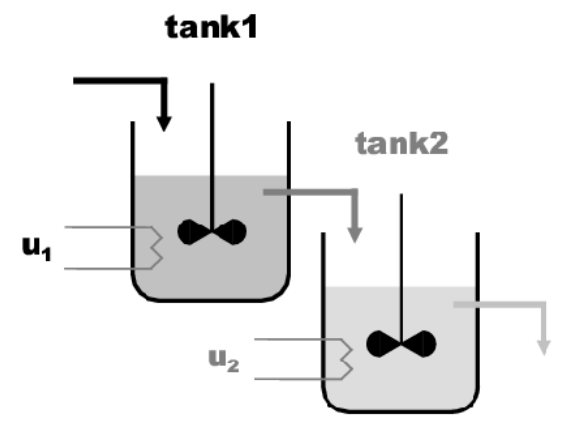

Fig. 1. 2 stages CSTR

scribed by the following equations.

$$
\left\{\begin{aligned}
\dot{x}_{1}=-\left(1-\Delta_{1}(t)\right) x_{1}-R_{1}+a \\
\dot{x}_{2}=-2 x_{2}+R_{1}-a+\Delta_{2}(t)-\left(x_{2}+a^{2}\right) u_{1} \\
\dot{x}_{3}=-x_{3}+\left(1+\Delta_{3}(t)\right) x_{1}(t-\tau(t))-R_{2}+a^{2} \\
\dot{x}_{4}=-2 x_{4}+x_{2}(t-\tau(t))+R_{2}+\Delta_{4}(t)-a^{2} \\
\quad-\left(x_{4}+a^{2}\right) u_{2}
\end{aligned}\right.
$$

where $x_{1}$ and $x_{3}$ are normalized concentration variables in tank 1 and 2 , respectively, and $x_{2}$ and $x_{4}$ are respectively normalized temperature variables in tank 1 and 2 . The reaction rate term $R_{1}$ and $R_{2}$ in tank 1 and tank 2 are given by

$$
R_{1} \triangleq\left(x_{1}+a\right) e^{\left(\frac{25 x_{2}}{x_{2}+2}\right)}, \quad R_{2} \triangleq\left(x_{3}+a^{2}\right) e^{\left(\frac{25 x_{4}}{x_{4}+2}\right)},
$$

and $a=1 / 2$. Moreover $\Delta_{i}(i=1, \ldots, 4)$ denote perturbations satisfying $\left|\Delta_{i}(t)\right|<d_{i}, i=1, \ldots, 4$, and $\tau(t)$ is a time-varying delay whose bound is given by $[0,0.5]$. For the system, we consider the following nominal model (Oh and Luus, 1976).

$$
\left\{\begin{array}{l}
\dot{x}_{1}=-x_{1}-R_{1}+a \\
\dot{x}_{2}=-2 x_{2}+R_{1}-a-\left(x_{2}+a^{2}\right) u_{1} \\
\dot{x}_{3}=-x_{3}+x_{1}(t-\ell)-R_{2}+a^{2} \\
\dot{x}_{4}=-2 x_{4}+x_{2}(t-\ell)+R_{2}-a^{2}-\left(x_{4}+a^{2}\right) u_{2}
\end{array}\right.
$$

where $\ell$ is a constant delay. Then according to (12), the perturbation vector $p(t)$ is given by

$$
p(t)=\left(\begin{array}{c}
\Delta_{1}(t) x_{1} \\
\Delta_{2}(t) \\
\left(\Delta_{3}(t)+1\right) \tilde{\sigma} x_{1}-\sigma x_{1} \\
\Delta_{4}(t)+\tilde{\sigma} x_{2}-\sigma x_{2}
\end{array}\right)
$$

where $\tilde{\sigma}: \lambda(t) \rightarrow \lambda(t-\tau(t))$ and $\sigma: \lambda(t) \rightarrow$ $\lambda(t-\ell)$. For the nominal model (22), there exist $\bar{\phi}_{1}(x)$ and $\bar{\phi}_{2}(x)$ satisfying condition (i)-(iv) for $x_{2}, x_{4}>-a^{2}$. Therefore, by applying a variable transformation $z(t)=\Phi(x, \boldsymbol{\sigma})$ as

$$
z(t)=\left(\begin{array}{c}
x_{1} \\
-x_{1}-R_{1}+a \\
x_{3} \\
-x_{3}+\sigma x_{1}-R_{2}+a^{2}
\end{array}\right)
$$

and feedback (4), we obtain

$$
\frac{d}{d t}\left(\begin{array}{l}
z_{1}^{i} \\
z_{2}^{i}
\end{array}\right)=\left(\begin{array}{ll}
0 & 1 \\
0 & 0
\end{array}\right)\left(\begin{array}{l}
z_{1}^{i} \\
z_{2}^{i}
\end{array}\right)+\left(\begin{array}{l}
0 \\
1
\end{array}\right) v_{i}+p_{m}^{i}+p_{u m}^{i}
$$

for $i=1,2$, where $p_{m}^{i} \triangleq \operatorname{col}\left(0, L_{p} L_{\bar{f}} \bar{\phi}_{i}\right)$ and $p_{u m}^{i} \triangleq \operatorname{col}\left(L_{p} \bar{\phi}_{i}, 0\right)$. Now, by choosing a sliding surface as $S_{i}\left(z^{i}\right)=s_{i 1} z_{1}^{i}+z_{2}^{i}$ for $i=1,2$, a control law is decided by

$$
\left\{\begin{array}{l}
v_{i}(t)<-s_{i 1} z_{2}^{i}-M_{i}, \text { if } S_{i}\left(z_{i}\right)>0 \\
v_{i}(t)>-s_{i 1} z_{2}^{i}+M_{i}, \text { if } S_{i}\left(z_{i}\right)<0
\end{array}\right.
$$

where $M_{i} \geq \sup _{p}\left|s_{i 1} L_{p} \bar{\phi}_{i}(x, \boldsymbol{\sigma})+L_{p} L_{\bar{f}} \bar{\phi}_{i}(x, \boldsymbol{\sigma})\right|$. By applying a switching input satisfying (24), the system arrives at the sliding surface, the dynamics of the system in the sliding mode is described by the following equation.

$$
\frac{d}{d t}\left(\begin{array}{l}
z_{1}^{1} \\
z_{2}^{1}
\end{array}\right)=\left(\begin{array}{cc}
-s_{11} & 0 \\
0 & -s_{21}
\end{array}\right)\left(\begin{array}{l}
z_{1}^{1} \\
z_{1}^{2}
\end{array}\right)+\Delta(t)
$$

where

$$
\Delta(t) \triangleq\left(\begin{array}{c}
L_{p} \bar{\phi}_{1} \\
L_{p} \bar{\phi}_{2}
\end{array}\right)=\left(\begin{array}{c}
\Delta_{1}(t) \cdot x_{1} \\
\Delta_{3}(t) \cdot \sigma x_{1}
\end{array}\right) .
$$

Here $\Delta(t)$ satisfies the following inequality.

$$
\begin{aligned}
\|\Delta(t)\| & =\sqrt{\Delta_{1}^{2} x_{1}^{2}+\Delta_{3}^{2} \cdot \sigma x_{1}^{2}} \\
& \leq \sqrt{d_{1}^{2}+d_{3}^{2}} \sup _{\theta \in[-\ell, 0]}\left|x_{1}(t+\theta)\right| \\
& \leq \sqrt{d_{1}^{2}+d_{3}^{2}} \sup _{\theta \in[-\ell, 0]}\left\|\left(\begin{array}{c}
\bar{\phi}_{1}(t+\theta) \\
\bar{\phi}_{2}(t+\theta)
\end{array}\right)\right\|
\end{aligned}
$$

Therefore if we choose $s_{11}$ and $s_{21}$ satisfying $s_{i}>\sqrt{d_{1}^{2}+\left(d_{3}+2\right)^{2}}, z$ converges to zero and in turn $x$ also converges to zero. Figure 2, 3 and 4 show the behaviour of the state variables $z(t)$ and $x(t)$ and the inputs $u_{1}$ and $u_{2}$, respectively. In the simulation, the time-varying delay was given by $\tau(t)=0.5 \sin 10 \pi t$ and the bounds of the perturbations were by $d_{1}=d_{3}=0.5, d_{2}=0.02$ and $d_{4}=0.04$. Then $s_{11}$ and $s_{21}$ were selected as $s_{11}=s_{21}=2.6$.

\section{CONCLUSION}

In this paper, we have derived a sufficient condition to guarantee robust stability for uncertain retarded nonlinear systems with time-varying delay by using the Lyapunov-Razumikhin approach. By using the derived criterion, we can successfully design a sliding mode controller to stabilize retarded nonlinear systems with uncertainties and time-varying delay. 


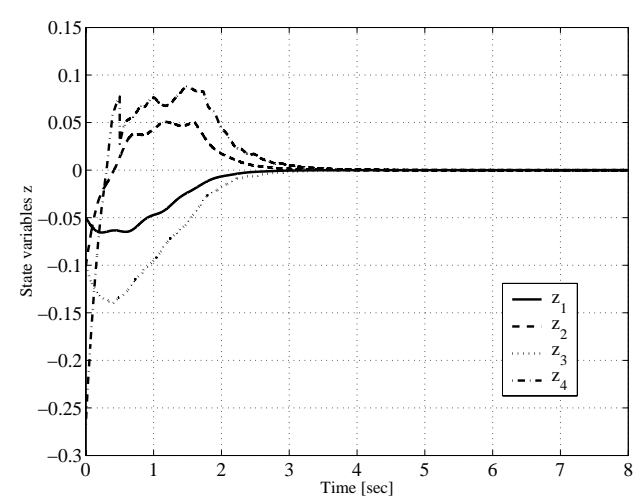

Fig. 2. Behaviour of $z(t)$

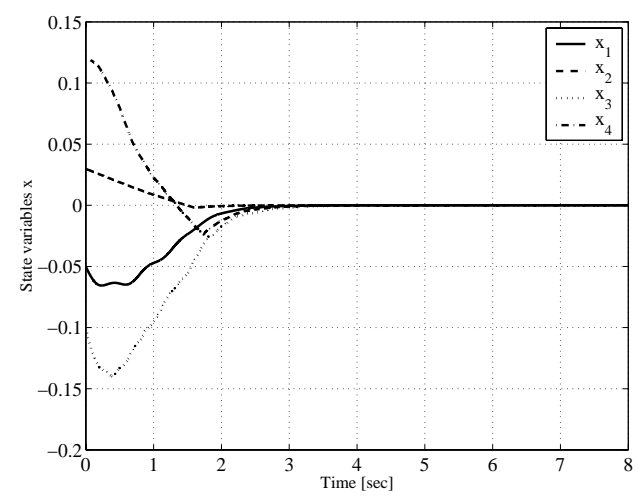

Fig. 3. Behaviour of $x(t)$
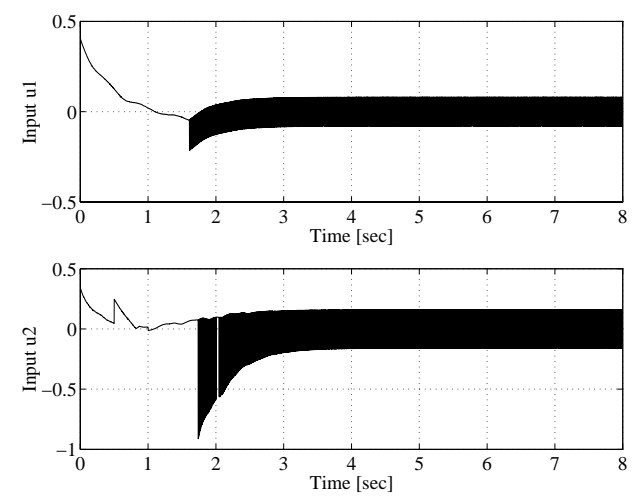

Fig. 4. Inputs $u_{1}(t)$ and $u_{2}(t)$

\section{REFERENCES}

Bonnet, C., J. Partington and M. Sorine (1999). Robust control and tracking of a delay system with discontinuous non-linearity in the feedback. Int. J. Control 72(15), 1354-1364.

Cheres, E., S. Gutman and Z. Palmor (1989). Stabilization of uncertain dynamic systems including state delay. IEEE Trans. Automat. Contr. 34(11), 1199-1203.

Choi, H. (1999). An LMI approach to sliding mode control design for a class of uncertain time delay systems. In: Proc. of European Control Conference. Karlsruhe, Germany.

El-Khazaly, R. (1998). Variable structure robust control of uncertain time-delay systems. Automatica 34(3), 327-332.
Gouaisbaut, F., M. Dambrine and J.-P. Richard (2002). Robust control of delay systems: A sliding mode control design via LMIs. System 8 Control Letters 46, 219-230.

Isidori, A. (1995). Nonlinear Control Systems - An Introduction. 3rd ed.. Springer-Verlag.

Li, X. and S. Yurkovich (1999). Sliding Mode Control of Systems with Delayed State and Controls. Vol. 247. Springer-Verlag.

Luo, N. and M. De la Sen (1993). State feedback sliding mode control of a class of uncertain time-delay systems. IEE proceedingsD 140(4), 261-274.

Morse, A. S. (1976). Ring models for delaydifferential systems. Automatica 12, 529-531.

Niculescu, S. I. (2001). Delay effects on stability A Robust Control Approach. Springer.

Nijmeijer, H. and A. van der Schaft (1990). Nonlinear Dynamical Control Systems. SpringerVerlag.

Oguchi, T., A. Watanabe and T. Nakamizo (1999). Finite spectrum assignment for nonlinear systems with non-commensurate delays. In: Proc. of 14th IFAC World Congress. Vol. C. pp. 85-90.

Oguchi, T. and A. Watanabe (2000). Finite spectrum assignment for multivariable retarded nonlinear systems. In: Proc. CD of the 14 th MTNS. pp. 999-1004.

Oguchi, T., S. Kawata and J.-P. Richard (2002). Sliding mode control of retarded nonlinear systems via finite spectrum assignment approach. In: Proc. of the 41st IEEE Conference on Decision and Control. Las Vegas. pp. 9991004.

Oh, S. H. and R. Luus (1976). Optimal feedback control of time-daly systems. AIChE Journal 22(1), 140-147.

Perruquetti, W. and J. Barbot (2002). Sliding Mode Control for Engineers. Marcel Dekker.

Shyu, K. and J. Yan (1993). Robust stability of uncertain time-delay systems and its stabilization by variable structure control. Int. J. Control 57, 237-246.

Sira-Ramirez, H. (1988). Differential geometric methods in variable-structure control. Int. J. Control 48(4), 1359-1390.

Sira-Ramirez, H. (1989). Nonlinear variable systems in sliding mode: The general case. IEEE Trans. Automat. Contr. 34(11), 1186-1188.

Slotine, J.E. and W. Li (1996). Nonlinear Systems. 2nd ed.. Prentice-Hall.

Sontag, E. D. (1976). Linear systems over commutative rings: A survey. Ricerche di Automatica $7(1), 1-34$.

Utkin, V. (1992). Sliding Modes in Control Optimization. Springer-Verlag.

Young, K. D. and Özgüner, Ü., Eds.) (1999). Variable Structure Systems, Sliding Mode and Nonlinear Control. Springer. 